\title{
Research on the Application Value of BIM in Building Construction
}

\author{
Duan Shixia $^{1, \mathrm{a}}$, Jin Yangliu ${ }^{2, \mathrm{~b}}$ \\ ${ }^{1}$ School of Management Engineering, Zhengzhou University, Zhengzhou, China \\ ${ }^{2}$ School of Management Engineering, Zhengzhou University, Zhengzhou, China
}

\begin{abstract}
In order to adapt to the rapid development of the national economic level, as well as to meet people's higher and higher requirements for the living environment, the construction industry must break the traditional extensive management of the construction model, and introduce new science and technology into building construction. Based on the three objectives of project management, including schedule, cost and quality control, this paper points out the limitations of project management under traditional construction methods, and points out that the application of BIM technology represents the latest direction of modern information technology application in the engineering construction industry. By studying the impact of BIM technology on traditional construction and highlighting the value of applying BIM technology in the field of construction, it is expected to find a construction management mode more suitable for modern construction enterprises, so as to promote the sustainable development of the construction industry.
\end{abstract}

\section{INTRODUCTION}

BIM technology, namely Building Information Modeling, is a kind of digital Information technology vigorously promoted by the state at present ${ }^{[1]}$. It converts digital Information into Building models to show the actual situation of the project. It is characterized by visualization, coordination, simulation, optimization and cartography. According to data from the National Bureau of Statistics (NBS), the GDP of the construction industry nationwide in 2019 was 2.48446 trillion yuan, accounting for $25.07 \%$ of THE GDP ( 99086.5 billion yuan), with a year-on-year growth of $5.7 \%$. The construction industry is still the pillar industry of the national economy. Construction development depends largely on the level of construction, although with the development of national economy of our country construction level got bigger, but compared with developed countries there is still a big gap, the traditional construction technology can't meet the present stage in the process of engineering construction projects in the construction requirements. A significant change in information and communication technology in the construction industry is that BIM, as a new computeraided design, has been widely applied in the industry and academia ${ }^{[2]}$.

Starting from the meaning and characteristics of BIM technology, Shan Lin conducted in-depth exploration and analysis ${ }^{[3]}$. Taking a large-scale public construction project as an example, Zhang Jian et al. demonstrated the application value of BIM technology in the design stage by introducing the main work content and application scope of BIM technology in the design stage $\mathrm{e}^{[4]}$. Liu Hui et al. summarized and sorted out the cutting-edge literature of BIM internationally, and explored the value of BIM in construction engineering innovation according to the category of construction engineering innovation ${ }^{[5]}$. Wen Quan et al. studied the value of BIM application in the whole process of green building by means of literature analysis and questionnaire survey, and constructed the theoretical framework of BIM application value evaluation ${ }^{[6]}$. BIM technology as at present the country vigorously promote digital information technology and its application in the field of building construction, to the very positive impact on the existing construction technology level, including increasing the accuracy of the expression of the construction site conditions, improve project management level and effect, such as the use of BIM technology in the field of building construction makes the traditional extensive management of the construction process of slowly shift towards the direction of the fine management, not only conforms to the current national policy guide, but also to the inevitable trend of the present construction industry sustainable development.

\section{COMPARISON OF CONSTRUCTION METHODS BEFORE AND AFTER APPLICATION OF BIM TECHNOLOGY}

\subsection{Main problems existing in traditional construction methods}

There are two traditional methods of construction schedule planning: the transverse chart method and the

$\overline{\text { aduanshixia@sina.com, }{ }^{b} 18838969155 @ 163 . c o m}$ 
engineering network plan chart. Under the traditional construction method, cost control mainly USES various organizational, economic, contract and other measures to adjust the cost of people, materials, machinery, and project subcontracting involved. Under the traditional construction method, quality control mainly follows the PDCA cycle principle, that is, the continuous cycle of planning, implementation, inspection, disposal and four operations; three - stage control principle and total quality management principle.

The main problems of traditional schedule control are as follows: design itself has defects; the construction schedule is not flexible enough; coordination work, schedule control work difficult to cover all aspects. The main problems of the traditional cost control are as follows: the data processing ability related to the cost is poor; the ability to manage costs dynamically is poor. The main problems of traditional quality control are as follows: low skill level of project practitioners; improper selection of materials and machinery; it is easy to ignore the influence of external environment.

\subsection{Influence of BIM on traditional construction}

Compared with traditional construction methods, when BIM technology is applied in the field of building construction, the biggest advantage of BIM technology lies in the integration and application of information ${ }^{[7]}$. In the process control, BIM technology finds out the deficiencies of the design scheme in advance and optimizes it by simulating the construction site. In cost control, the role of BIM technology is mainly reflected in that BIM model can be adjusted at any time according to the actual situation, so as to truly achieve dynamic management and monitoring of project cost. In quality control, the role of BIM is mainly reflected in the networked management and sharing of data and information, which facilitates project management personnel to make timely decisions to prevent quality accidents. In addition, errors in construction drawings can be found timely through simulated construction, and construction drawings can be deepened to reduce the quality accident rate.

To sum up, the influence of BIM technology on building construction can be summarized as follows: auxiliary construction scheme simulation and optimization, auxiliary digital processing, dynamic schedule cost management and monitoring, auxiliary drawing joint review, auxiliary technology disclosure, and auxiliary construction drawing deepening.

\section{ANALYSIS OF ADVANTAGES AND DISADVANTAGES OF CONSTRUCTION METHODS BEFORE AND AFTER THE APPLICATION OF BIM TECHNOLOGY}

To reasonable assessment and application effect of BIM following the introduction of construction technology, combined with the construction sector has the characteristics of strong fuzzy evaluation factors, using grey correlation degree and fuzzy comprehensive evaluation method, quantitative and qualitative evaluation of the construction site indexes of construction method before and after the introduction of BIM analyzing two cases, and identify the importance of introducing BIM technology in the field of building construction.

\subsection{Improved grey relational degree method to determine the weight of evaluation factors}

\subsubsection{Improve the grey relational degree method}

Scholars Cui Jie and Dang Yaoguo et al. found that the grey relational degree method has certain defects ${ }^{[8]}$. First of all, there are too many factors affecting the weight result. For example, the selection of reference value $x_{0}(k)$ and the value of resolution coefficient $\rho$ will affect the operation result of correlation coefficient $\xi_{i}(k)$, thus affecting the calculation result of weight. Secondly, the weight of the distinction is small. Therefore, this paper USES the grey relational degree improvement method proposed by Cui Jie, Dang Yao Guo and other scholars. Based on expert experience scoring method, evaluation indexes are obtained and evaluation matrix is formed. The maximum value is selected as the common reference value to form the reference sequence. The distance between each index and the reference sequence is obtained. The weight can be further solved by normalization.

In order to compare and analyze the advantages and disadvantages of the construction methods before and after the application of BIM technology, an evaluation factor set needs to be established. Based on the six influence points of BIM technology extracted on building construction, the evaluation factor set of "the advantages and disadvantages of construction methods before and after the application of BIM technology" is determined as $\mathrm{U}=\{$ Simulation and optimization of construction scheme, digital processing, dynamic schedule cost management and monitoring, joint review of drawings, technical disclosure, deepening of construction drawings $\}$.

\subsubsection{Expert scoring method to determine the judgment value}

Set $B=\{B 1, B 2, B 3, B 4, B 5, B 6\}=U$ as the set of evaluation factors. Five experts with two years or more experience in BIM application were invited to score the six evaluation factors according to likert's five-point scale.

TABLE I. EXPERT SCORING TABLE

\begin{tabular}{|c|c|c|c|c|c|}
\hline A & expert 1 & expert 2 & expert 3 & expert 4 & expert 5 \\
\hline B1 & 4 & 3 & 3 & 3 & 3 \\
\hline B2 & 2 & 3 & 2 & 3 & 3 \\
\hline B3 & 4 & 4 & 4 & 5 & 4 \\
\hline B4 & 3 & 4 & 3 & 4 & 4 \\
\hline
\end{tabular}




\begin{tabular}{|l|l|l|l|l|l|}
\hline B5 & 3 & 2 & 1 & 3 & 2 \\
\hline B6 & 4 & 3 & 5 & 4 & 5 \\
\hline
\end{tabular}

\subsubsection{Calculate the weight of influencing factors by} grey relational degree analysis

3.1.3.1 According to the degree of influence of factors table, form a judgment matrix form a judgment matrix $X$.

$$
X=\left(\begin{array}{cccc}
x_{1}(1) & x_{1}(2) & \cdots & x_{1}(5) \\
x_{2}(1) & x_{2}(2) & \cdots & x_{2}(5) \\
\cdots & \cdots & \cdots & \cdots \\
x_{6}(1) & x_{6}(2) & \cdots & x_{6}(5)
\end{array}\right)
$$

3.1.3.2 Find out the maximum influence degree $x_{0}(k)$ from the judgment matrix, take it as the reference value, assign this value to each influence degree, thus forming a new matrix $X^{\prime}$.

$$
X^{\prime}=\left(\begin{array}{cccc}
x_{0}(k) & x_{0}(k) & \ldots & x_{0}(k) \\
x_{1}(1) & x_{1}(2) & \ldots & x_{1}(5) \\
x_{2}(1) & x_{2}(2) & \ldots & x_{2}(5) \\
\cdots & \ldots & \ldots & \ldots \\
x_{6}(1) & x_{6}(2) & \cdots & x_{6}(5)
\end{array}\right)
$$

3.1.3.3 Use the formula $D_{i}=\sum_{k=1}^{5}\left[x_{0}(k)-x_{i}(k)\right]^{2}$, get the distance value $D_{i}$.

3.1.3.4 From $W_{i}=\frac{1}{1+D_{i}}$, the weight of each factor is $W_{i}$.

3.1.3.5 The weight value obtained is normalized to obtain the final weight result $W_{i}^{\prime}=(0.1034,0.0600$, $0.3723,0.1551,0.0433,0.2659)$.

In order to facilitate the calculation and retain two decimal points, Six influencing factors of BIM technology on building construction: construction scheme simulation and optimization, digital processing, dynamic management and monitoring of schedule and cost, joint review of drawings, technical disclosure and deepening of construction drawings have value weights of $0.10,0.06$, $0.37,0.16,0.04$ and 0.27 respectively.

\subsection{Comparison of construction methods before and after the introduction of BIM by fuzzy comprehensive evaluation method}

\subsubsection{Invite experts to grade and determine the evaluation matrix}

The construction process of the construction project is divided into four evaluation grades: excellent, good, medium and poor. Five experts with two years or more experience in the BIM application field are invited to evaluate and score the effects of the six evaluation factors in the construction process according to the four grades. The scoring results are shown in Table II and Table III respectively.

TABLE II. TRADITIONAL CONSTRUCTION METHODS EXPERT GRADING TABLE

\begin{tabular}{|c|c|c|c|c|}
\hline \multirow{2}{*}{ The evaluation factors } & \multicolumn{4}{|c|}{$\begin{array}{c}\text { Select the number of experts } \\
\text { at each evaluation level }\end{array}$} \\
\cline { 2 - 5 } & $\begin{array}{c}\text { excelle } \\
\boldsymbol{n} \boldsymbol{t}\end{array}$ & $\begin{array}{c}\text { goo } \\
\boldsymbol{d}\end{array}$ & $\begin{array}{c}\text { mediu } \\
\boldsymbol{m}\end{array}$ & poor \\
\hline $\begin{array}{c}\text { Simulation and optimization of } \\
\text { construction scheme }\end{array}$ & 0 & 0 & 1 & 4 \\
\hline Digital processing & 0 & 0 & 3 & 2 \\
\hline $\begin{array}{c}\text { Dynamic schedule cost } \\
\text { management and monitoring }\end{array}$ & 0 & 1 & 2 & 2 \\
\hline Joint review of drawings & 0 & 2 & 2 & 1 \\
\hline Technical disclosure & 0 & 1 & 3 & 1 \\
\hline $\begin{array}{c}\text { Deepening of construction } \\
\text { drawing }\end{array}$ & 0 & 0 & 3 & 2 \\
\hline
\end{tabular}

TABLE III. EXPERT GRADING TABLE AFTER THE INTRODUCTION OF BIM

\begin{tabular}{|c|c|c|c|c|}
\hline \multirow{2}{*}{ The evaluation factors } & \multicolumn{4}{|c|}{$\begin{array}{c}\text { Select the number of experts at } \\
\text { each evaluation level }\end{array}$} \\
\cline { 2 - 5 } & excellent & good & medium & poor \\
\hline $\begin{array}{c}\text { Simulation and optimization } \\
\text { of construction scheme }\end{array}$ & 2 & 3 & 0 & 0 \\
\hline Digital processing & 4 & 1 & 0 & 0 \\
\hline $\begin{array}{c}\text { Dynamic schedule cost } \\
\text { management and monitoring }\end{array}$ & 3 & 2 & 0 & 0 \\
\hline Joint review of drawings & 1 & 3 & 1 & 0 \\
\hline Technical disclosure & 1 & 2 & 2 & 0 \\
\hline $\begin{array}{c}\text { Deepening of construction } \\
\text { drawing }\end{array}$ & 1 & 3 & 1 & 0 \\
\hline
\end{tabular}

Let the evaluation set be $\mathrm{V}=$ \{excellent, good, medium, poor $\}=\{\mathrm{v} 1, \mathrm{v} 2, \mathrm{v} 3, \mathrm{v} 4\}$. The single-factor evaluation

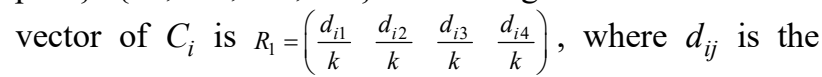
number of votes to evaluate the ith factor $C_{i}$ as $V_{j}$ of the $j$ level, and $k$ is the total number of experts. Let the evaluation matrix under the traditional construction method be $R_{1}$ and the evaluation matrix after applying BIM be $R_{2}$. According to the expert scoring results, it can be calculated as follows: 


$$
R_{1}=\left(\begin{array}{cccc}
0 & 0 & 0.2 & 0.8 \\
0 & 0 & 0.6 & 0.4 \\
0 & 0.2 & 0.4 & 0.4 \\
0 & 0.4 & 0.4 & 0.2 \\
0 & 0.2 & 0.6 & 0.2 \\
0 & 0 & 0.6 & 0.4
\end{array}\right) \quad R_{2}=\left(\begin{array}{cccc}
0.4 & 0.6 & 0 & 0 \\
0.8 & 0.2 & 0 & 0 \\
0.6 & 0.4 & 0 & 0 \\
0.2 & 0.6 & 0.2 & 0 \\
0.2 & 0.4 & 0.4 & 0 \\
0.2 & 0.6 & 0.2 & 0
\end{array}\right)
$$

\subsubsection{Comparison of construction methods before and after the introduction of BIM}

The weights of the six evaluation factors are $\mathrm{A}=\{$ construction plan simulation and optimization, digital processing, dynamic schedule cost management and monitoring, joint review of drawings, technical disclosure, construction drawing deepening $\}=\{0.10,0.06,0.37,0.16$, $0.04,0.27\}$. According to the operation rules of $B=A R$, then:

$$
B_{1}=A R_{1}=\left(\begin{array}{llllll}
0.10 & 0.06 & 0.37 & 0.16 & 0.04 & 0.27
\end{array}\right)\left(\begin{array}{llll}
0 & 0 & 0.2 & 0.8 \\
0 & 0 & 0.6 & 0.4 \\
0 & 0.2 & 0.4 & 0.4 \\
0 & 0.4 & 0.4 & 0.2 \\
0 & 0.2 & 0.6 & 0.2 \\
0 & 0 & 0.6 & 0.4
\end{array}\right)=\left(\begin{array}{llll}
0 & 0.146 & 0.454 & 0.4
\end{array}\right)
$$

And get $B_{2}=\left(\begin{array}{llll}0.404 & 0.494 & 0.102 & 0\end{array}\right)$ in the same way.

After calculation, the fuzzy comprehensive evaluation results of the building construction process before and after the introduction of BIM technology can be obtained as shown in Table IV.

TABLE IV. FUZZY COMPREHENSIVE EVALUATION RESULTS BEFORE AND AFTER BIM IS INTRODUCED INTO THE CONSTRUCTION PROCESS

\begin{tabular}{|c|c|c|c|c|}
\hline \multirow{2}{*}{$\begin{array}{c}\text { The introduction } \\
\text { of BIM }\end{array}$} & \multicolumn{4}{|c|}{ Evaluation percentage (\%) } \\
\cline { 2 - 5 } & excellent & good & medium & poor \\
\hline $\begin{array}{c}\text { Before the } \\
\text { introduction of BIM }\end{array}$ & 0 & 14.6 & 45.4 & 40.0 \\
\hline $\begin{array}{c}\text { After the } \\
\text { introduction of BIM }\end{array}$ & 40.4 & 49.4 & 10.2 & 0 \\
\hline
\end{tabular}

It can be seen from Table 4 that after the application of BIM technology, the excellent and good evaluation rates of all evaluation factors have been improved to some extent. After the introduction of BIM technology by construction enterprises, the evaluation rate of excellence and good in the whole construction process has risen from $14.6 \%$ to $89.8 \%$, indicating that BIM technology occupies an absolute advantage in the application of building construction field. Therefore, it is very necessary to apply BIM technology in the construction process.

\subsection{Conclusion of the value of BIM technology in building construction}

Fuzzy comprehensive evaluation results show that BIM technology can greatly improve the excellent and good rate of construction process when it is applied to building construction, which indicates that BIM technology has advantages that traditional construction methods do not have and can make the whole construction project more perfect in the implementation process. When BIM technology is applied in building construction, its biggest characteristic is its strong ability to process information, including faster collection of information, more accurate storage of information, more timely transmission of information, and more efficient analysis and application of information. In view of the efficient integration and processing capacity of BIM technology for information, compared with traditional construction methods, the advantages of BIM technology after its introduction into building construction can be summarized as follows:

(1) Strong ability of building information expression and digital processing.

(2) Find design loopholes and optimize the design plan in advance of simulated construction.

(3) Integrate relevant in formation in time to realize the dynamic management and monitoring of the project.

(4) Facilitate the timely communication and transmission of relevant information among all parties involved in the construction project.

Compared with the traditional construction method, BIM technology has obvious advantages in the construction process, and its application value in the construction is very great, indicating that the practice of introducing BIM technology in the construction field is very desirable and worth advocating. Combined with the advantages of BIM technology in building construction, several application values of BIM technology in building construction are summarized as follows:

(1) Virtual construction, optimization scheme.

(2) Fast calculation, improve the accuracy.

(3) Precise planning to reduce waste.

(4) Share information and collaborate effectively.

The application value of BIM technology in building construction indicates the application of information technology in the field of building construction, and indicates the development direction of modern construction industry to combine with information technology for common progress.

\section{CONCLUSION}

Based on the three control objectives of project management, namely schedule, cost and quality, this paper firstly analyzes the deficiency of the three management modes under the traditional construction methods. Secondly, the influencing factors of BIM technology on traditional construction are summarized. Then, the grey relational grade method and fuzzy comprehensive evaluation method, comparative analysis before and after the application of BIM technology construction methods, summed up the BIM technology are introduced into the construction of the advantages, highlight the BIM technology application to the application value in the field of building construction, and the construction should be combined with modern information technology is obtained the conclusion of common development. 


\section{REFERENCES}

1. Feng Yufu. Application of BIM Technology in construction Project Construction[J]. Sichuan building materials, 2018,44(08):216+222.

2. David Bryde,Martí Broquetas,Jürgen Marc Volm. The project benefits of Building Information Modelling (BIM) $[\mathrm{J}]$. International Journal of Project Management,2013,31(7).

3. Shan Lin. Application of BIM Technology in construction Engineering project [J]. Modern Property Management (Mid-Jun.),2020(04):56-57.

4. Zhang Jian, Huang Jie, Su Taoyong. Application Value Analysis of BIM in the design of large-scale public construction projects [J].Building science,2019,35(01):45-50.

5. Liu hui, wang mengjun,Miroslaw J.Skibniewski, zheng junwei. Preliminary exploration on the innovative value of BIM in construction engineering projects $[\mathrm{J}]$. Science and technology management research,2016,36(08):194-200.

6. Wen Quan, LI Zhongfu, LI Zhouyang, Li Tianxin.Research on the Application Value Evaluation System of BIM in the whole process of green building $[\mathrm{J}]$. Journal of Engineering Management,2020(04): 1-7.

7. Peng Hao, Qiu Gang. Application value of BIM Technology in construction Engineering projects [J]. Residential and Real Estate, 2018(02):191.

8. Cui Jie, Dang Yao-guo, Liu Si-feng.An Improved method for Solving index Weight based on Grey Relational Degree[J]. China Management Science,2008 (05):141-145. 